SLAC-PUB-9127

January 2002

\title{
Black Hole Production at the LHC: Effects of Voloshin Suppression *
}

\author{
Thomas G. Rizzo \\ Stanford Linear Accelerator Center \\ Stanford University \\ Stanford CA 94309, USA
}

\begin{abstract}
We examine the rates for the production of black holes(BH) at the LHC in light of the exponential suppression of the geometric cross section estimate recently proposed by Voloshin. We show that the resulting production rates will still be quite large over a reasonably wide range of model parameters. While $\mathrm{BH}$ production may no longer be the dominant collider process, its unique signature will ensure observability over more conventional backgrounds.
\end{abstract}

*Work supported by the Department of Energy, Contract DE-AC03-76SF00515 
Theories with extra dimensions and a low effective Planck scale $\left(M_{*}\right)$ offer the exciting possibility that the production rate of black holes(BH) somewhat more massive than $M_{*}$ can be quite large at future colliders. For example, cross sections of order $100 \mathrm{pb}$ at the LHC[1], and even larger ones at the VLHC, have been advertised in the analyses presented by Giddings and Thomas(GT) and by Dimopoulos and Landsberg(DL). Although in practice the actual production cross section critically depends on the BH mass, the exact value of $M_{*}$ and the number of extra dimensions, following the analysis of the authors in Ref.[1], one finds very large rates over almost all of the interesting parameter space. These earlier analyses and discussions of the production of $\mathrm{BH}$ at colliders have been elaborated upon by several groups of authors[2] and the production of $\mathrm{BH}$ by cosmic rays has also been considered[3]. The most important question to address is whether or not the BH cross sections are actually this large or, at the very least, large enough to lead to visible rates at future colliders.

The basic idea behind the original collider BH papers is as follows: we consider the collision of two high energy Standard Model(SM) partons which are confined to a 3-brane, as they are in both the models of Arkani-Hamed, Dimopoulos and Dvali(ADD)[4] and Randall and Sundrum(RS)[5]. In addition, we imagine that gravity is free to propagate in $\delta$ extra dimensions with the $4+\delta$ dimensional Planck scale assumed to be $M_{*} \sim 1 \mathrm{TeV}$. The curvature of the space is assumed to be small compared to the energy scales involved in the collision process so that quantum gravity effects can be neglected. When these partons have a center of mass energy in excess of $\sim M_{*}$ and the impact parameter for the collision is less than the Schwarzschild radius, $R_{S}$, associated with this center of mass energy, a $4+\delta$-dimensional $\mathrm{BH}$ is formed with reasonably high efficiency. It is expected that a very large fraction of the collision energy, i.e., $\sqrt{s}$, actually goes into the $\mathrm{BH}$ formation process so that $M_{B H} \simeq \sqrt{s}$. The subprocess cross section for the production of a non-spinning $\mathrm{BH}$ is thus essentially 
geometric for each pair of initial partons:

$$
\hat{\sigma} \simeq \epsilon \pi R_{S}^{2}
$$

where $\epsilon$ is an factor that accounts for finite impact parameter and angular momentum corrections and which is expected to be $\lesssim 1$. (We will assume that $\epsilon=1$ in our calculations below.) We note that the $4+\delta$-dimensional Schwarzschild radius, $R_{S}$, scales as

$$
R_{S} \sim\left[\frac{M_{B H}}{M_{*}^{2+\delta}}\right]^{\frac{1}{1+\delta}}
$$

apart from an overall $\delta$ - and convention-dependent numerical prefactor. This convention dependence has been found to be somewhat confusing for unprepared readers of the literature and particularly to experimenters investigating the BH production phenomena. The confusion can be traced back to two different expressions for the $4+\delta$ dimensional Schwarzschild radius used by the original two sets of authors GT and DL. Explicitly, the two sets of authors employed distinct relationships between their $4+\delta$-dimensional Planck masses, $M_{G T, D L}$, and the associated generalized Newton's constant, $G_{4+\delta}$ : one finds that $M_{D L}^{2+\delta}=G_{4+\delta}^{-1}$, while $M_{G T}^{2+\delta}=(2 \pi)^{\delta} / 4 \pi G_{4+\delta}$. Depending on how the input parameters are chosen, i.e., which of the above masses is identified with $M_{*}$, this scheme dependent prefactor can turn out to be relatively important. In particular, it is found to lead to an apparently very different $\delta$-dependence for the $\mathrm{BH}$ production cross section in the two cases. In the DL case the $\delta$-dependence of the numerical prefactor is rather weak whereas is it somewhat stronger in the GT analysis. Naively, for the same input value of $M_{B H}$ one finds the ratio of the cross sections obtained by the two sets of authors to be

$$
\frac{\hat{\sigma}_{G T}}{\hat{\sigma}_{D L}}=\left[\frac{(2 \pi)^{\delta}}{(4 \pi)}\right]^{\frac{2}{1+\delta}}\left[\frac{M_{D L}^{2}}{M_{G T}^{2}}\right]^{\frac{2+\delta}{1+\delta}},
$$

which is always greater than unity for $\delta \geq 2$ and grows as $\delta$ increases if one assumes $M_{*}=$ $M_{G T}=M_{D L}$ as an input. (The later equality is, of course, invalid.) It is important to note, 
however, that when the differences in the definitions of the Planck scale are accounted for, both cross sections lead to the same numerical results. This can be accomplished by using a common value of $G_{4+\delta}$ as input in both cases. Written in terms of $G_{4+\delta}$ the above ratio of cross sections is unity. Unfortunately, since $G_{4+\delta}$ is a rather cumbersome object to use as an input parameter, in our discussion below we will display results for both sets of notation, i.e., we will identify $M_{*}$ as either $M_{D L}$ or $M_{G T}$.

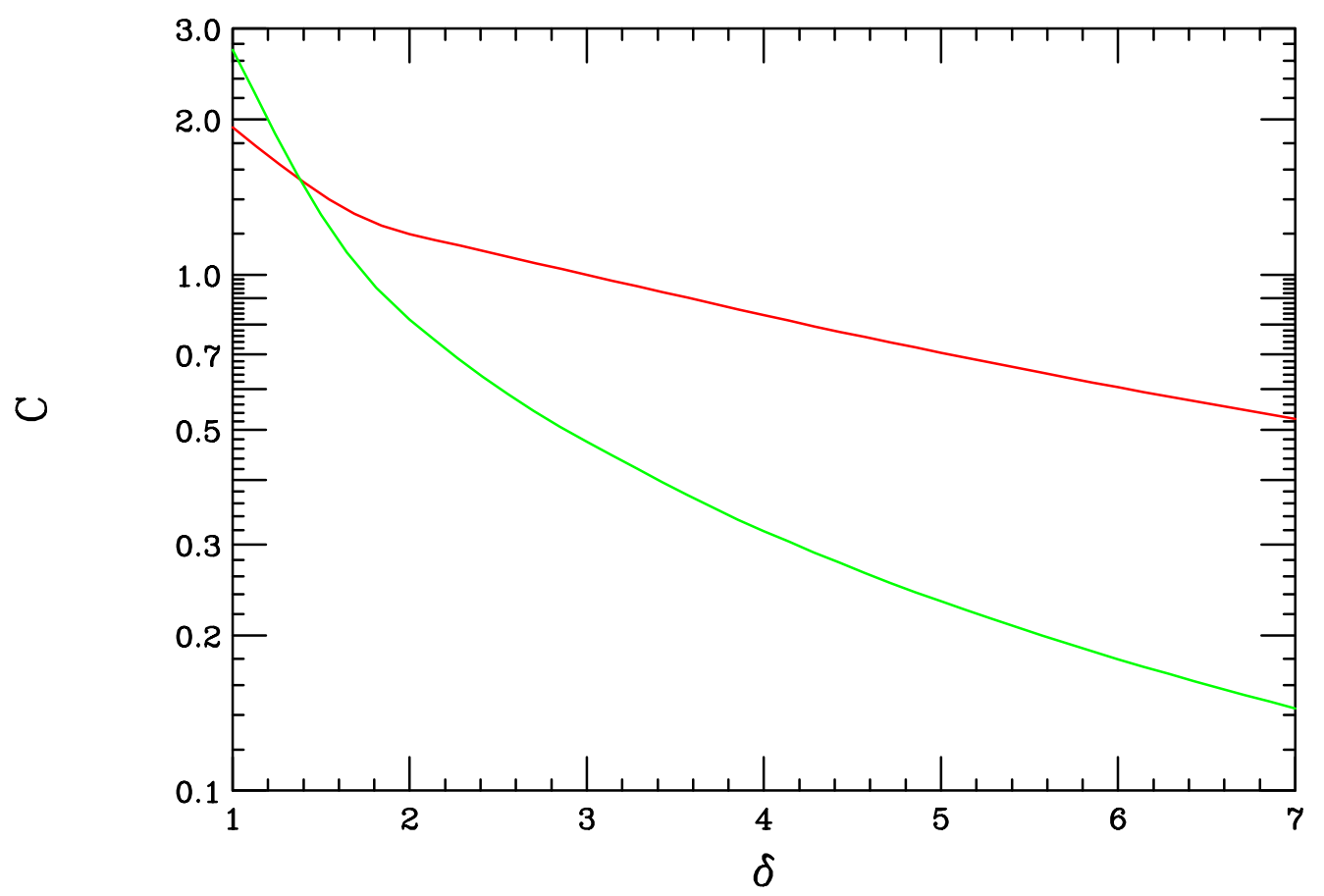

Figure 1: The parameter $C$ appearing in the exponential suppression factor of Voloshin as a function of the number of extra dimensions, $\delta$. The upper(lower) curve on the right-hand side of the plot is for the GT(DL) scheme.

The approximate geometric subprocess cross section expression is claimed to hold by both GT and DL when the ratio $M_{B H} / M_{*}$ is "large", i.e., when the system can be treated semi-classically and quantum gravitational effects are small; one may debate just what "large" really means, but it most likely means "at least a few". Certainly when $M_{B H} / M_{*}$ is near unity one might expect curvature and stringy effects to become important and even the finite extent of the incoming partons associated with this stringy-ness would 
need to be considered. Clearly caution must be applied when $M_{B H} \simeq M_{*}$ in interpreting cross sections evaluated in this parameter space region.

In order to obtain the actual cross section at a collider, one takes the geometric parton-level result, folds in the appropriate parton densities, sums over all pairs of possible partons and then integrates over the relevant kinematic variables. The resulting total cross section for $\mathrm{BH}$ with masses $\geq M_{B H}^{m i n}$ is then given by the expression

$$
\sigma=\int_{M_{B H}^{\min 2} 2 / s}^{1} d \tau \int_{\tau}^{1} \frac{d x}{x} \sum_{a b} f_{a}(x) f_{b}(\tau / x) \hat{\sigma}\left(M_{B H}\right)
$$

where we have summed over all possible pairs of initial state partons with their associated densities $f_{i}(x)$. These parton densities are evaluated at a scale $Q^{2}=M_{B H}^{2}$ in the numerical results presented below.

Voloshin has recently[6] provided several arguments which suggest that an additional exponential suppression factor, $S=e^{-I_{E}}$, must be included which seriously damps the pure geometric cross section for this process even in the semi-classical case. This implies we should rescale the purely geometric result as $\hat{\sigma} \rightarrow S \hat{\sigma}$ in the equations above. Here, $I_{E}$ is the Euclidean Gibbons-Hawking action for the $\mathrm{BH}[7]$ generalized to the case of $4+\delta$ dimensions. Then $S$ is given explicitly by

$$
S=\exp \left[-4 \pi R_{S} M_{B H} /(1+\delta)(2+\delta)\right]=\exp \left[-C\left(\frac{M_{B H}}{M_{*}}\right)^{\frac{2+\delta}{1+\delta}}\right]
$$

where $C$ is a relatively small, though $\delta$-dependent, constant whose detailed form is also dependent upon whether the GT or DL schemes are followed. Fig. 1 shows the value of $C$ as a function of $\delta$ for both schemes. In the $\mathrm{DL}(\mathrm{GT})$ scheme, for example, when $\delta=3$ one obtains $C=0.474(0.999)$; similarly for $\delta=5$ one obtains $C=0.233(0.706)$. Note that in either scheme we see that $C$ is rather small and decreases substantially as $\delta$ is increased. 

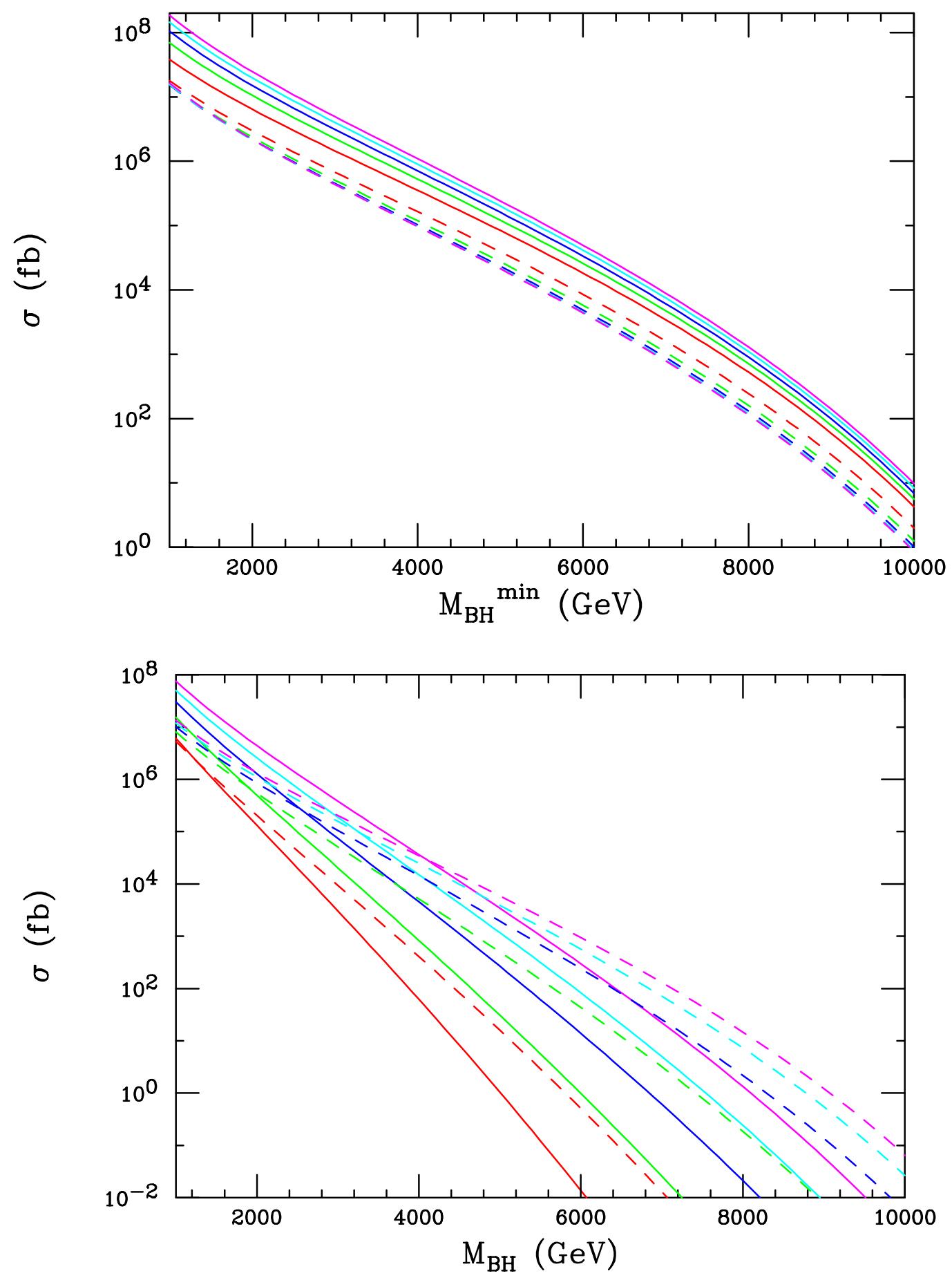

Figure 2: (Top)Cross section for the production of BH more massive than $M_{B H}^{\min }$ at the LHC assuming $M_{*}=1 \mathrm{TeV}$ for $\delta=2(3,4,5,6)$ extra dimensions with the solid(dashed) curves showing the results by Giddings and Thomas(Dimopoulos and Landsberg). In the GT(DL) case an increase in the number of extra dimensions leads to an increase(decrease) of the cross section. We identify $M_{*}$ with either $M_{G T}$ or $M_{D L}$ for the appropriate set of curves. (Bottom)Same as the Top, but now including the effects of the Voloshin damping factor. We observe that large cross sections remain possible over a reasonable range of the model parameters. In both cases, DL or GT, the cross section now increases with the number of extra dimensions. 
Again, when the differences in notation in the two schemes are accounted for , i.e., using the same value of $G_{4+\delta}$, the value of the suppression factor is identical. The smallness of $C$ thus seriously reduces the numerical impact of the exponential suppression. From the $\delta$-dependence of $C$ and $\delta$-dependence of the power of the ratio $M_{B H} / M_{*}$ in the exponential, it is clear that the Voloshin suppression will become less effective as the number of extra dimensions increases.

While the possibility of the existence of exponential suppression remains controversial, and strong arguments have been made on either side of the argument, for purposes of this discussion we will assume that it is indeed present. (However, we warn the reader that the jury is still out on this issue. In either case we anxiously await the resolution of this important argument.) If Voloshin's criticisms of the geometrical cross section are valid one may certainly worry that the resulting exponentially suppressed rates for heavy $\mathrm{BH}$ production will possibly be too small to be observable at the LHC; as we will see below this need not be so. This is partly due to the smallness of $C$ and partly due to the expected huge size of the unsuppressed production rates for $\mathrm{BH}$ at the $\mathrm{LHC}$.

Just how do the suppressed and unsuppressed cross sections at the LHC compare? As can be seen in Fig. 2 for the case $M_{*}=1 \mathrm{TeV}$, the unsuppressed rates for $\mathrm{BH}$ production at the LHC are quite large over a wide range of masses and numbers of extra dimensions using either set of authors' cross section expressions. (It is important to remember that in this figure and the others below we appropriately identify $M_{*}$ as either $M_{G T}$ or $M_{D L}$ depending on which set of predictions are under discussion.) Note that the results of Giddings and Thomas always appear larger than those of Dimopoulos and Landsberg due to the different definitions used for the Planck scale and that these apparent differences between the two sets of predictions increases as $\delta$ increases as was discussed above. This result is of course just a simple artifact of the known notational difference. We also see that Fig. 2 shows 
the effects of the suppression predicted by Voloshin in the two cases. From these results we make the important observation that for at least for some ranges of parameters $\mathrm{BH}$ will still be produced at rates that are large enough to be observable at the LHC even when the Voloshin suppression is active. For example, assuming that $M_{B H}^{\min }=5 \mathrm{TeV}$, we see that cross sections can easily be in the 100-1000 fb range. Although this is not a huge cross section the associated rates at the LHC will be quite large given an integrated luminosity of order $100 \mathrm{fb}^{-1} / \mathrm{yr}$. Note that the Voloshin suppression factor apparently modifies the two sets of predictions in quite different manners due to the two different expressions used for $R_{S}$. Since $\left(R_{S}\right)_{G T}>\left(R_{S}\right)_{D L}$ for all $\delta \geq 2$, assuming the same input values for $M_{G T}$ and $M_{D L}$, the GT results are found to appear more suppressed than are those of DL. Again, if we take the notational differences in the two schemes into account, identical cross sections are obtained in both cases.

What happens as we vary $M_{*}$ ? Figs. 3 and 4 show the effects of increasing $M_{*}$ from $1 \mathrm{TeV}$ to 2 and $3 \mathrm{TeV}$, respectively. As expected the unsuppressed rates for any fixed value of $M_{B H}$ decreases but we also see that the Voloshin suppression becomes less effective. This is also to be expected since the ratio $M_{B H} / M_{*}$ in the exponent of the factor $S$ has been decreased for fixed $M_{B H}$. Again we see that for $\mathrm{BH}$ in the $5-6 \mathrm{TeV}$ range it is relatively likely that the production cross section can quite easily be in excess of $100 \mathrm{fb}$.

We remind the reader that once produced these BH essentially decay semi-classically, mostly on the brane, via Hawking radiation into a reasonably large number $\simeq 25$ or more final state partons with energies of order 50-100 GeV in a highly spherical pattern. Hadrons will be seen to dominate over leptons by a factor of order 5-10 for such final states. These unusual signatures would not be missed at either hadron or lepton colliders. (We note that an alternative decay scenario has been advocated by Casadio and Harms[8].) These features are sufficiently unique that BH production above conventional backgrounds should be observable 

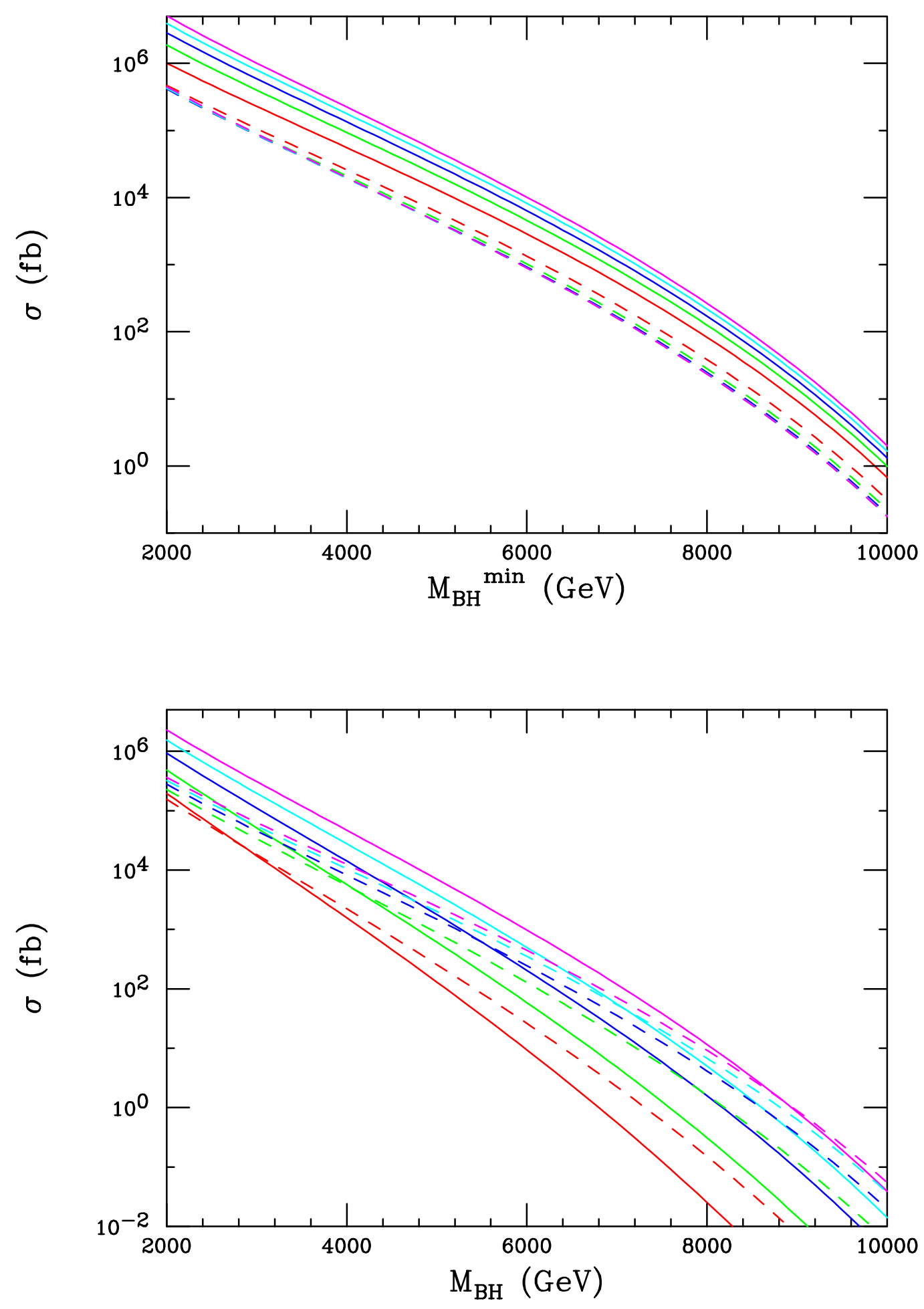

Figure 3: Same as the last figure but now for a larger value of the fundamental Planck scale, $M_{*}=2 \mathrm{TeV}$. 
at the LHC even if the cross sections are substantially smaller than the original estimates.

In summary, we have examined the production of BH at the LHC assuming that the exponential suppression of the geometric cross section predicted by Voloshin is realized. We have found that even when this suppression is significant the resulting $\mathrm{BH}$ production rates are still quite large for a wide range of model parameters given an integrated luminosity of order $100 \mathrm{fb}^{-1}$. If the scale $M_{*}$ is $\sim \mathrm{TeV}$ then $\mathrm{BH}$ production should provide an exciting signature at the LHC and open a door to even more exciting physics.

\section{Acknowledgements}

The author would like to thank S. Thomas, G. Landsberg and J. Hewett for discussions related to this work.

\section{References}

[1] S.B. Giddings and S. Thomas, hep-ph/0106219; S. Dimopoulos and G. Landsberg, Phys. Rev. Lett. 87, 161602 (2001).

[2] S.B. Giddings, hep-ph/0110127; K.Cheung, hep-ph/0110163; G. Landsberg, hepph/0112061; A. Ringwald and H. Tu, Phys. Lett. B525, 135 (2002); S. Hofmann et al., hep-ph/0111052; S.C. Park and H.S. Song, hep-ph/0111069; D.M. Eardley and S.B. Giddings, gr-qc/0201034; S. Hossenfelder, S. Hofmann and M. Bleicher, hep-ph/0109085. See also E.-J. Ahn, M. Cavaglia and A.V. Olinto, hep-th/0201042.

[3] M. Kowalski, A. Ringwald and H. Tu, hep-ph/0201139; L.A. Anchordoqui, J.L. Feng, H. Goldberg and A.D. Shapere, hep-ph/0112247; Y. Uehara, hep-ph/0110382; R. Emparan, M. Masip and R. Rattazzi, hep-ph/0109287; J. Alfaro and G. Palma, hep-th/011176; 

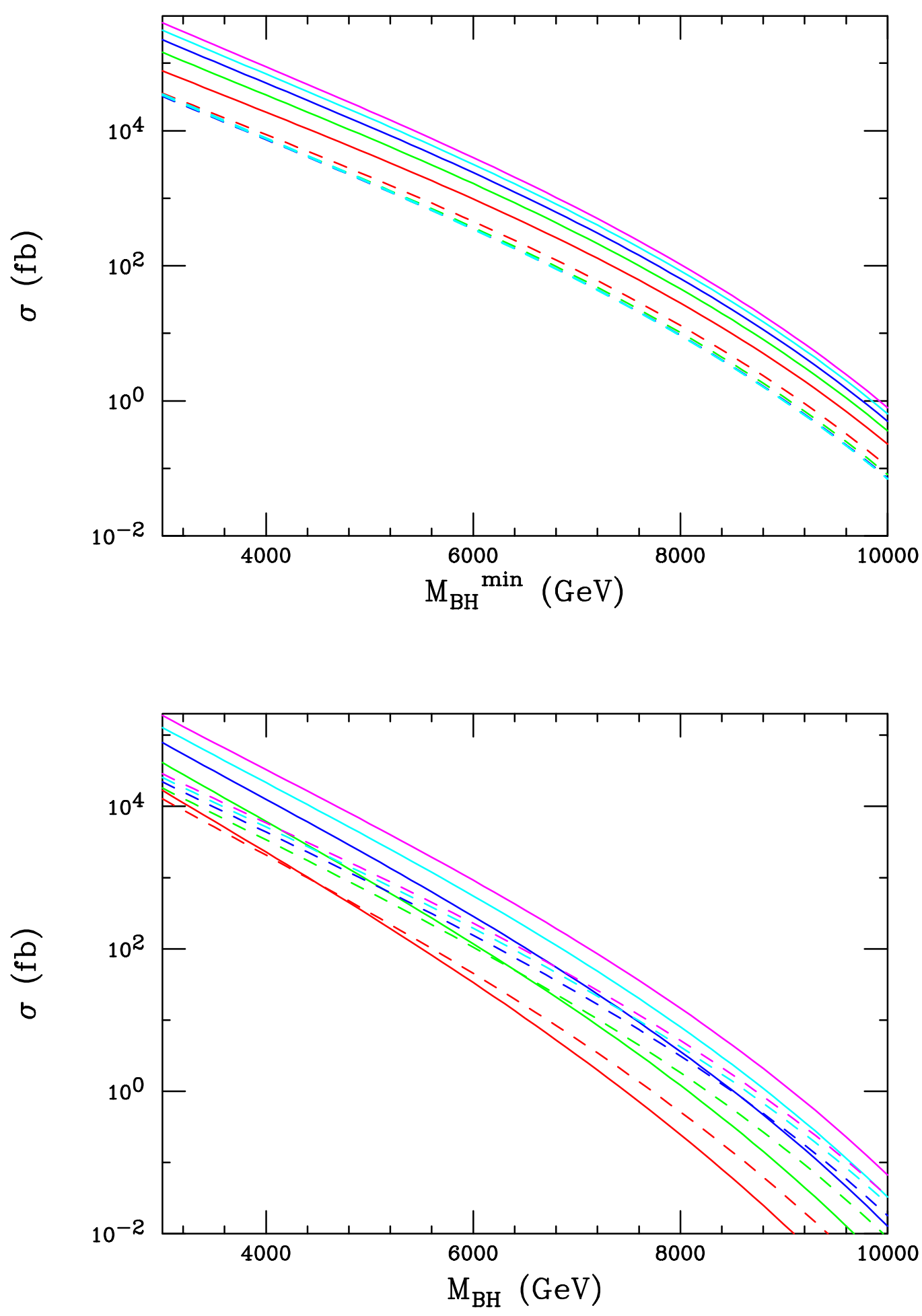

Figure 4: Same as the last figure but now for $M_{*}=3 \mathrm{TeV}$. 
D. Kazanas and A. Nicolaidis, hep-ph/0109247; L.A. Anchordoqui and H. Goldberg, hep-ph/0109242; J.L. Feng and A.D. Shapere, Phys. Rev. Lett. 88, 021303 (2002).

[4] N. Arkani-Hamed, S. Dimopoulos, and G. Dvali, Phys. Lett. B429, 263 (1998), and Phys. Rev. D59, 086004 (1999); I. Antoniadis, N. Arkani-Hamed, S. Dimopoulos, and G. Dvali, Phys. Lett. B436, 257 (1998).

[5] L. Randall and R. Sundrum, Phys. Rev. Lett. 83, 3370 (1999).

[6] M.B. Voloshin, Phys. Lett. B518, 137 (2001) and Phys. Lett. B524, 376 (2002 ;)see, however, the arguments presented in S. Dimopoulos and R. Emparan, hep-ph/0108060.

[7] G.W. Gibbons and S.W. Hawking, Phys. Rev. D15, 2752 (1977).

[8] R. Casadio and B. Harms, hep-th/0110255. 